\title{
A Comparison of Two- and Three-Dimensional Size Distributions in a Cellular Material
}

\author{
P. L. WHITE ${ }^{a}$ AND L. H. VAN VLACK
}

This investigation concerns the comparison of two- and three-dimensional data obtained on a cellular material. By quantitative micrography techniques and spatial measurements, the cellular structure corresponds most closely with the shape of pentagonal dodecahedra, twelve-faced cells having five edges per face. The cell volumes have a normal distribution.

The areal distributions of planar sections for various shapes of polyhedra were taken from existing literature. The measured volume distributions from this study were then applied by numerical computer calculations to create a transformation which carried the distribution of volumes (three-dimensional) to the expected distribution of planar areas (two-dimensional). The results of the expected and measured areal data agreed well for assumed complex polyhedral symmetry such as pentagonal dodecahedra, and unsatisfactorily for spherical symmetry. These results demonstrate that the pentagonal dodecahedron is a measurable prototype of cell in grain shapes.

\section{Ein Vergleich von zwei- und dreidimensionalen Größenverteilungen an einem zellularen Stoff}

Die vorliegende Arbeit beschäftigt sich mit dem Vergleich von zwei- und dreidimensionalen Daten von Zellstrukturen. Mit Hilfe quantitativ mikroskopischer Untersuchungsverfahren und räumlichen Messungen wurde festgestellt, daß die Zellstruktur am besten durch die Form eines Pentagondodekaeders angenähert werden kann, d. h. einer Zelle mit 12 Flächen und 5 Kanten pro Fläche. Die Zellvolumina sind normal verteilt. Die Flächenverteilungen der ebenen Schnittflächen für verschiedene regelmäßige Polyeder wurden der vorhandenen Literatur entnommen. Die gemessenen Volumenverteilungen der vorliegenden Arbeit wurden dann einem Computer eingegeben, wobei die Volumenverteilungen (dreidimensional) in die erwarteten ebenen Verteilungen (zweidimensional) umgerechnet wurden. Die Ergebnisse der erwarteten und der gemessenen Flächenverteilungen stimmten gut mit der angenommenen komplexen polyedrischen Symmetrie, zum Beispiel der des Pentagondodekaeders überein, jedoch nur unbefriedigend mit der sphärischen Symmetrie. Diese Ergebnisse zeigen, daß das Pentagondodekaeder ein brauchbarer Prototyp für zellenförmige Körner ist.

a Dr. P. L. White is Senior Materials Scientist with Corporate Research, OwensIllinois Technical Center, Toledo, Ohio.

${ }^{b}$ Professor L. H. Van Vlack is Chairman, Chemical and Metallurgical Engineering Department, 'The University of Michigan, Ann Arbor, Michigan. 


\section{Une comparaison de la distribution des mesures à deux et à trois dimensions dans un matériau à structure cellulaire}

Cette étude est consacrée à la comparaison des mesures à deux et à trois dimensions qui ont été déterminées pour un matériau composé de cellules. D'après l'interprétation quantitative des micrographies ainsi que d'après des mesures spatiales, c'est à des dodécaèdres pentagonaux, donc à des cellules à douze faces ayant cinq arêtes par face, que la structure cellulaire correspond le mieux. Les volumes des cellules ont une distribution normale. Pour diverses formes de polyèdres, les distributions de surface de coupes planes ont été extraites de la littérature existant déjà. Les distributions volumétriques mesurées dans cette étude ont été soumises à un ordinateur pour les calculs numériques, afin de transposer la distribution volumétrique (à trois dimensions) en une distribution de surface (à deux dimensions) que l'on désirait déterminer. Les résultats des surfaces réellement mesurées correspondent bien aux données attendues pour la symétrie des polyèdres présumée complexe, donc par exemple pour les dodécaèdres pentagonaux; ils sont néanmoins insatisfaisants pour la symétrie sphérique. Ces résultats prouvent que le dodécaèdre polygonal est un prototype de cellule mesurable pour les formes des grains.

a Le Dr. P. L. White est "Senior Materials Scientist" au centre technique d'OwensIllinois, Toledo, Ohio.

b Le Professeur L. H. Van Vlack est président du service "Chemical and Metallurgical Engineering" de l'université du Michigan, Ann Arbor, Michigan.

\section{Introduction}

The opacity of most materials along with the inability to determine volumes of cells or grains accurately has led to numerous attempts to calculate particle or grain size distributions from measured planar data. For the most part, such calculations are based on an a priori assumption that all grains are spherical. While this is known to be incorrect, such symmetry simplifies the calculation of volume distributions. Efforts to achieve similar probabilities from planar intersections of more complex shapes has been largely neglected because of the mathematical difficulties.

Through the use of reticulated polyurethane foams (foams with only a skeletonal structure, having cell walls removed), however, it is possible to determine the cell volume distribution by individually measuring a statistically meaningful number of cells. In addition, the cellular structure of the foam can be defined in terms of orientation, faces, edges, and dimensions. Such a material also permits the observation of planar sectioning distributions by connecting straight lines between grain junctions and subsequently measuring the areas of each grain.

Thus a knowledge of the two-dimensional areal distribution plus the threedimensional volume distribution provides a direct check of various conversions from one distribution to the other. In addition, the structure may be classified as to complex polyhedral shapes, and transformations based on the complex 
symmetry may be carried out numerically with the aid of the computer. Therefore, it was feasible to make analyses of two- and three-dimensional distributions and to arrive at conclusions as to the validity of planar-spatial transformations based on assumed average shape, whether spherical symmetry was employed or not.

\section{Determination of Statistical Grain Size}

The shape and size of biological cells and metal grains has been under investigation since the seventeenth century. The three-dimensional shapes of biological cells have been reviewed by Lewis ${ }^{1,2}$ and Marvin. ${ }^{3}$ Marvin $^{4}$ also demonstrated that, when lead balls of uniform diameter are compressed so that the interstices are eliminated, the balls are changed into polyhedra averaging 14 faces, and that when small and large shot are mixed and compressed, the small balls are changed into polyhedra with fewer than 14 faces and the large ones into polyhedra having more than 14 faces.

Matzke $^{5}$ and Matzke and Nestler ${ }^{6}$ made intensive analyses of three-dimensional shapes of bubbles in foams. Bubbles of uniform volume had an average of 13.7 faces. Matzke found a predominance of pentagonal faces and concluded that pentagonal dodecahedra might be expected to be the most common shape, approximating the equilibrium conditions of $120^{\circ}$ angles between films and angles of $109^{\circ} 28^{\prime} 16^{\prime \prime}$ between edges. ${ }^{7}$

Desch $^{8}$ was able to examine the shapes of grains of beta-brass where there were 11 to 20 faces per grain with a predominance of five edges per face. Scheil and Wurst ${ }^{9}$ determined the spatial distribution of grain sizes in a sample of ingot iron by polishing off successive layers and following each grain through micrographs, deriving a statistical grain shape in terms of a calculated plane distribution curve, resulting in a distribution somewhat different from that obtained from a sphere.

Williams and Smith, ${ }^{10}$ using an $\mathrm{Al}-5.2 \%$ Sn alloy in which the tin concentrated in triangular areas at the grain corners, delineated a total of 91 grains through stereoscopic photographs which were taken by microradiography. That study indicated an average of five edges per face and of 12.48 faces per grain.

When the requirements for space filling, along with the minimization of surface-tension forces, are considered, Lord Kelvin ${ }^{11}$ demonstrated that a volume composed of truncated octahedra will completely fill space with a minimum of surface area. A polyhedron of this type, better known as the tetrakaidecahedron, is shown in Fig. 1. This 14-faced volume has six four-edged faces along with eight hexagonal faces and meets the surface tension requirements of no more than three grains at an edge and no more than four edges at a corner. A slight double curvature of the eight hexagonal faces satisfies the $120^{\circ}$ dihedral angle requirement for three adjacent grains meeting at an edge. Such curvature can be demonstrated in simple soap films. ${ }^{12}$ 


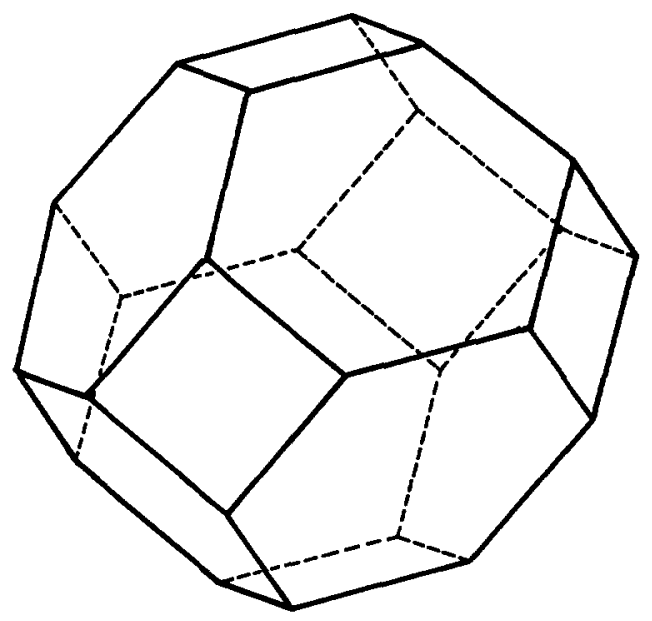

FIG. 1. Tetrakaidecahedron.

Desch ${ }^{8}$ pointed out in his study that both foam cells and grains approximated the shape of regular pentagonal dodecahedra. This polyhedral shape is shown in Fig. 2. It has 12 faces, all being pentagons, with the faces meeting at close to $120^{\circ}$ and the edges meeting at angles of $108^{\circ}$. Hence, small curvatures of the faces would bring this figure into conformity with surface tension requirements. Complete filling of space cannot be accomplished by stacking of pentagonal dodecahedra; but where polyhedra of varying shape are packed together

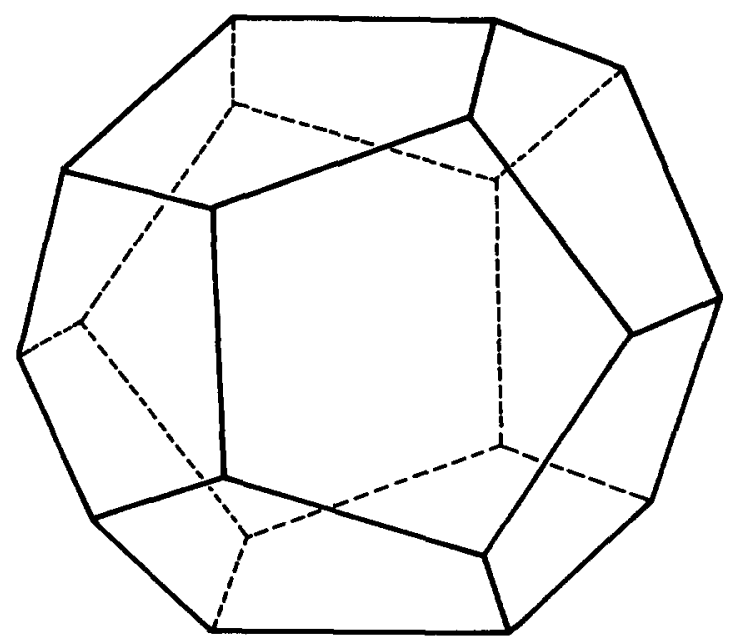

FIg. 2. Pentagonal dodecahedron. 
subject only to limitations on junctions of edges and grains, it is apparent that pentagonal dodecahedra may be frequently observed.

An additional limitation to that of filling space is that the shape of grains or cells must tend toward that of minimum surface energy. Because of the variation of shapes observed in nature, the closest prototype is the pentagonal dodecahedron. ${ }^{13}$ Most strictly the physical conditions comply with the viewpoint of Williams and Smith; ${ }^{10}$ - namely, that the individual grain is not governed by any one particular shape but conforms to the tetrahedral junction of four grains whose angles approximate $109^{\circ} 28^{\prime}$.

\section{Shapes of Grains in Two Dimensions}

Desch $^{8}$ and Hull and Houk ${ }^{14}$ have studied the two-dimensional shape of planar sections by using models of specific polyhedra. Myers ${ }^{15}$ has refined these results through computer calculations. It can be shown that six is the average number of sides when three grain boundaries meet at every corner, satisfying surface tension requirements. ${ }^{16}$

\section{Distribution of Grain Sizes in Three Dimensions}

Seldom, if ever, is a mass composed of identically sized grains. Rather a wide variation of volumes exists, as well as grain shapes. Furthermore, it can be shown that a wide range of sizes would be observed on a planar section even if all grains were the same size. ${ }^{14}$

Many studies have been devoted to calculate the volume distribution of particle or grain sizes from information obtained from planar sectioning data. Such methods are too lengthy to be discussed in detail, but are generally based on chord, diameter, or areal distributions, and usually assume spherical shape. Excellent up-to-date reviews of particle size distributions and grain-size distributions have been made by Underwood ${ }^{17}$ and Schuckher, ${ }^{18}$ respectively.

Aaron et al. ${ }^{19}$ calculated three-dimensional grain-size distributions on samples previously examined by Williams and Smith. ${ }^{10}$ While direct comparisons could not be made between the calculated and measured sizes, a strong dependence of the number of grains per unit volume on the number of class intervals was found, and methods based on spheres were concluded to fit the direct measurements.

\section{Experimental Procedure}

Polymer foam was provided through the courtesy of the Scott Foam Division, ScottPaper Company. This foam is a polyester polyurethane formed as a condensation polymer. Following formation, the cell walls were removed, leaving the foam in a flexible, open-pore or reticulated state. Figure 3 shows the structure of such a foam having a nominal density of $2 \mathrm{lb} / \mathrm{ft}^{3}$. 


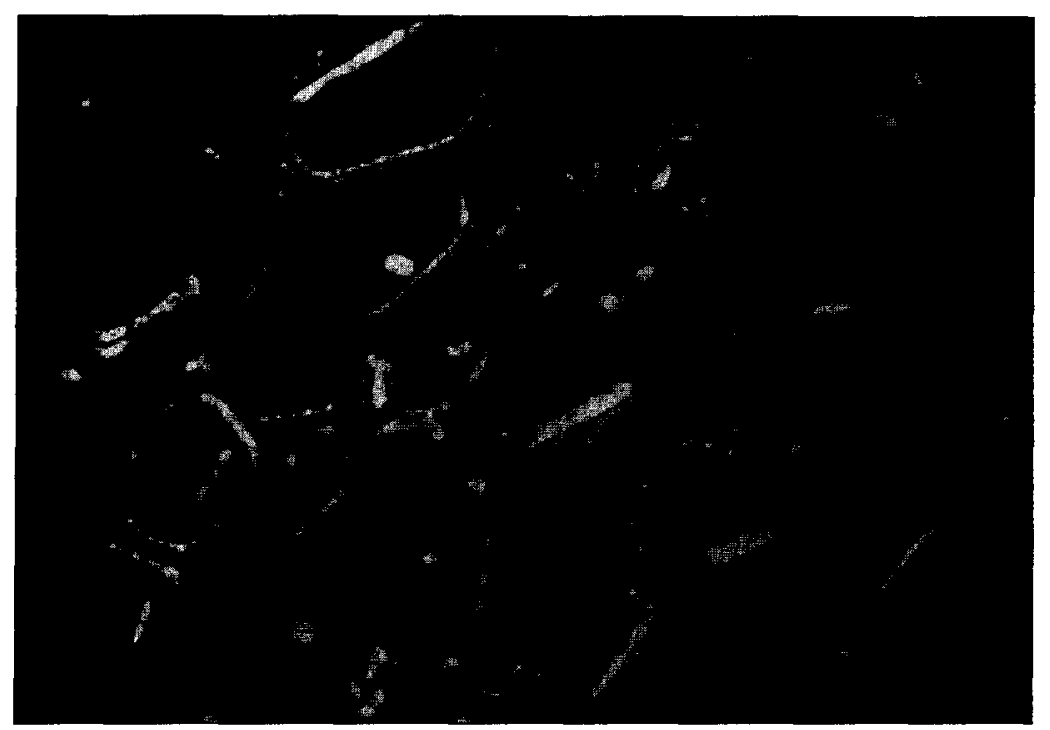

FIG. 3. Structure of 7-cell-per-inch polyurethane foam, density $2 \mathrm{lb} / \mathrm{ft}^{3} \cdot 6.2 \times$.

A cell volume distribution was obtained by filling individual cells from a 0.25 -cc syringe while observing the filling with a stereoscopic microscope. The amount of distilled water in the syringe was read before and after fill to $0.0025 \mathrm{cc}$. Filling was considered complete when the water "popped," wetting all members of the single cell. Figure 4 shows one individual cell within the foam, before filling, partially filled, and after filling. Measured variation of filling a number of individual cells repetitively resulted in a volume variation of $\pm 0.0025 \mathrm{cc}$. A total of 408 cells were individually measured for the volume distribution.

The filling of individual cells by surface tension resulted in planar surfaces for the cell faces. This method could result in a slight error in the volume distribution, but a planar face was desirable, since the two-dimensional areas were determined by straight-line intersection between second-phase material (see Fig. 5). Hence, a uniform comparison between two- and three-dimensional analyses was realized.

In addition to obtaining the volume distribution of cells within the structure, the number of faces of each of the polyhedra was also measured along with the corresponding number of edges per face. These two additional measurements aided in determining the geometrical shape of the "average" cell.

Foam samples were prepared for two-dimensional measurements by addition of a liquid polyester resin. Subsequent to solidification, samples were sectioned randomly and polished by hand. Pictures of the polished planar sections were taken on a Vickers projection microscope and on a stereoscopic zoom microscope.

Planar sections belonging to each individual cell were classed according to 


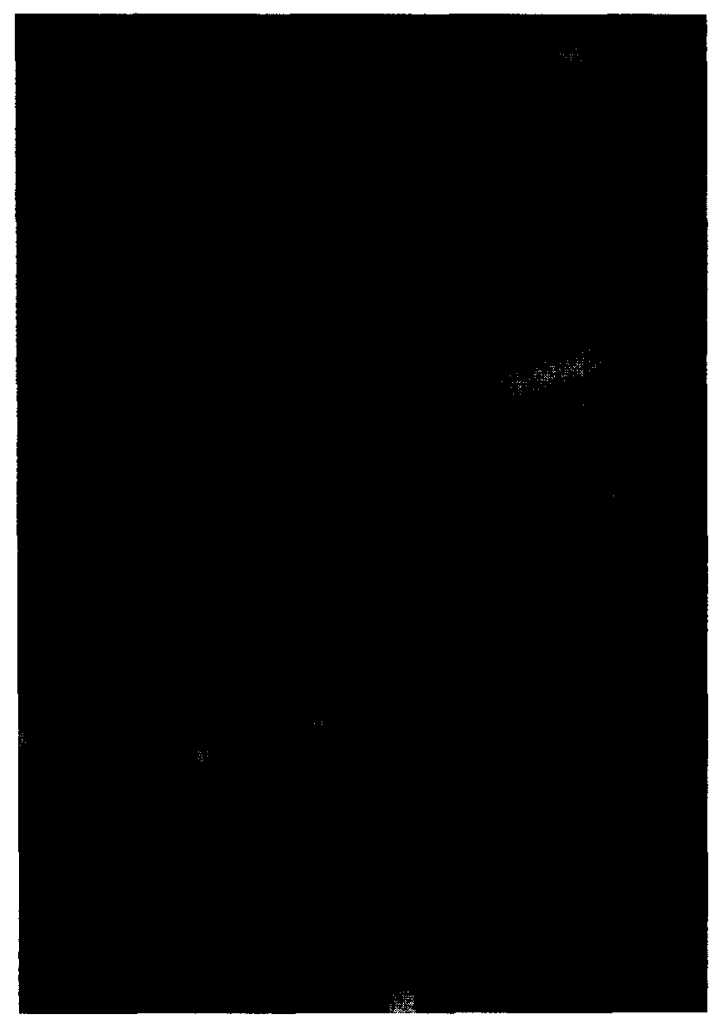

(a)

FIG. 4. Cell volume determination of polyurethane foam. $8.4 \times$. (a) Before filling.

their respective areas by direct comparison with circular templates having variation steps of $1 / 32$ inch in diameter above $3 / 8 \mathrm{inch}$, and 1/64 inch below. Areas were determined by drawing straight lines between the second-phase material. In this manner, planar sections were classed into 42 sizes ranging from 1 inch in diameter to $1 / 32$ inch in diameter, at a magnification of $4.2 \times$. The number of sides was counted for each planar cell section. Figure 5 shows the microstructure developed from infiltration of a foam specimen which has been prepared for areal analysis.

\section{Experimental Results and Discussion}

The measurements of the number of faces per foam cell demonstrate that the most probable number of faces is 14 with a mean of 13.2 faces per cell. No cells are observed with more than 17 or less than four faces, and statistically few cells have less than ten faces. The numbers of faces per polyhedron are 14 for 


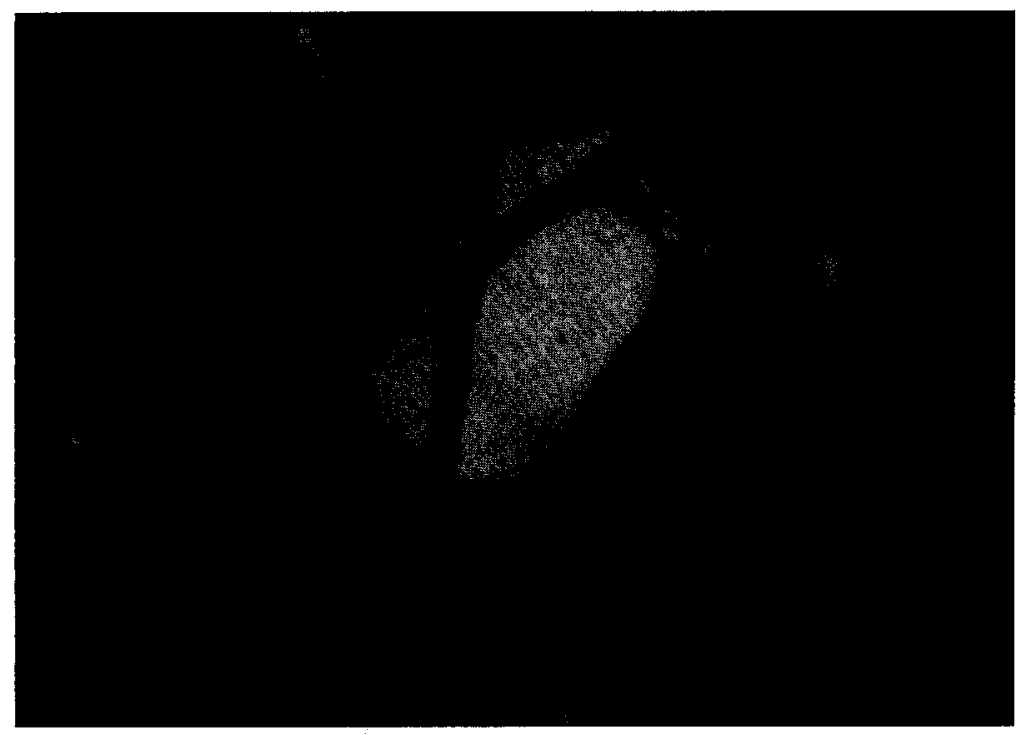

(b)

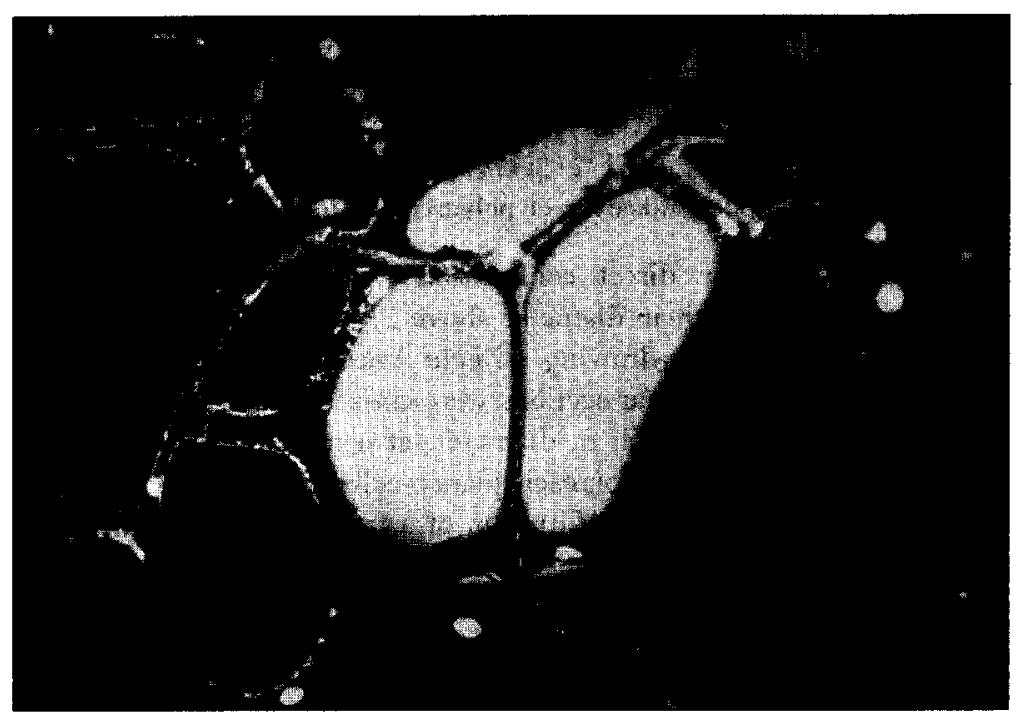

(c)

FIG. 4. (b) partially filled, volume $0.035 \mathrm{cc}$; (c) completely filled, volume $0.080 \mathrm{cc}$. 


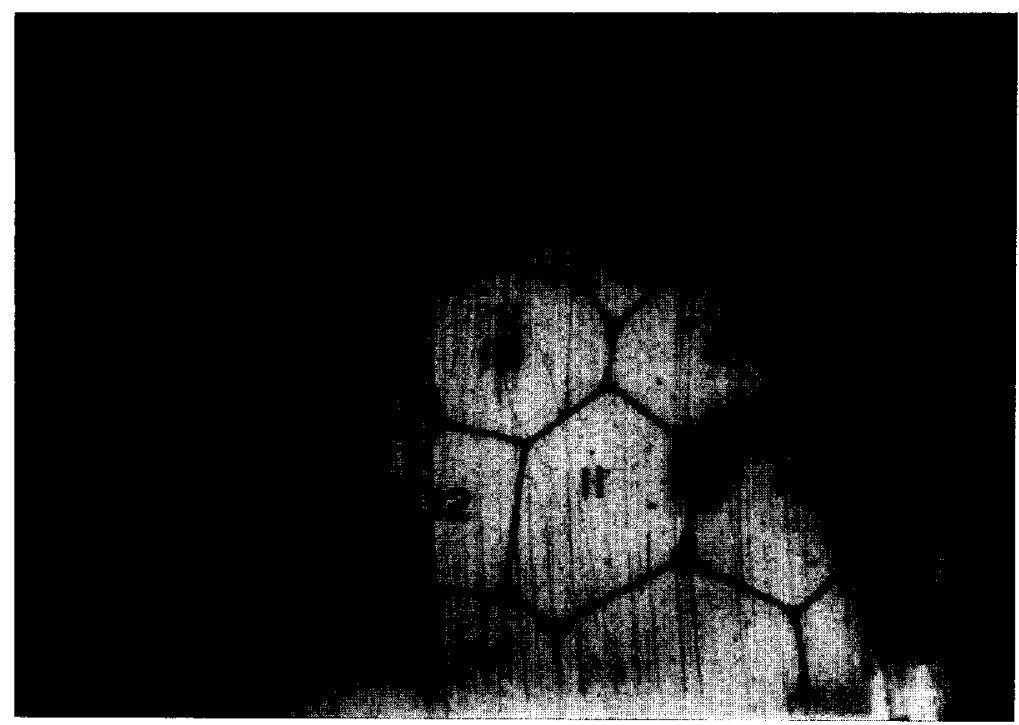

FIG. 5. Random planar section of foam prepared for quantitative analysis. $4.2 \times$.

the truncated octahedron (tetrakaidecahedron) and 12 for the pentagonal dodecahedron. Experimental observations on the shapes of cells in various materials show a strong similarity and confirm the general picture that the number of corners, edges, and faces observed are closely approximated by the averaged values of different asymmetrical polyhedra fitting together.

The distribution of faces per cell observed in this study is plotted in Fig. 6 along with the observed values of uniform interior bubbles and interior compressed lead shot. The observed distributions are quite similar, with 14 faces being predominate in all cases. For a further comparison, Table I shows the averaged results of measurements on froth bubbles, vegetable cells, and metallic grains. Five-edged faces are observed more than twice as often as any other in this particular foam structure. The mean of these results is 4.88 . Comparison with Table $I$ indicates that this figure may be low in relation to the number of faces per polyhedron, A further comparison with other observations is shown in Fig. 7, where the graphs exhibit the distribution of edges per face for the five different materials listed in Table I. The data measured in this study follow quite closely those observed for either mixed or uniform bubbles, but differ from those of metallic grains. The similarity to the bubbles should be expected because of the foam origin. Smith ${ }^{20}$ notes that foams owe their shape characteristics to the volumetrically insignificant part of matter lying in between the grains or cells, whereas metallic systems are influenced by the presence of nonmetallic inclusions and by the variation of grain boundary energy with direction and orientation. 


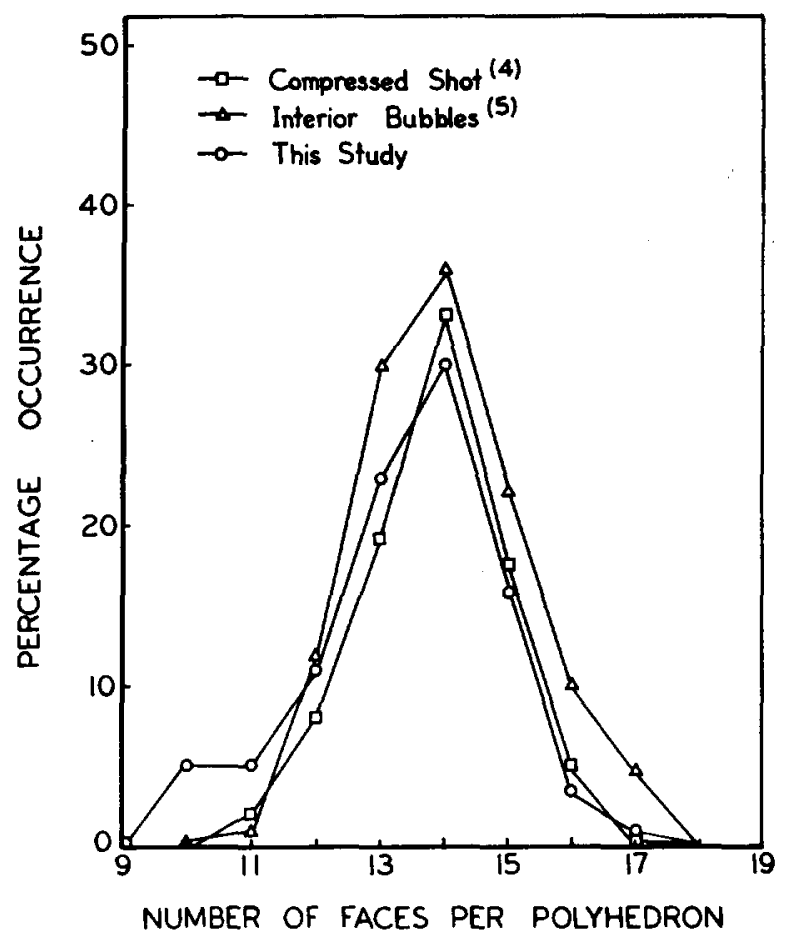

FIG. 6. Comparison of the distribution of number of faces per polyhedron for various materials.

Experimental observations of the frequency distribution of the number of edges or sides on plane sections in this study are compared with the distribution of edges per sectioned polygon for the pentagonal dodecahedron and the tetrakaidecahedron in Fig. 8. The average number of edges is 5.681 from this study,

TABLE I

Shapes of Bubeles, Cells, and Grains Considered as Separate Polyhedra

\begin{tabular}{lccc}
\hline & Edges/face & Faces/polyhedron & Reference \\
\hline 600 uniform bubbles & 5.111 & 13.702 & 5 \\
150 mixed bubbles & 5.095 & 13.260 & 6 \\
450 vegetable cells & 5.123 & 13.802 & 5 \\
30 beta-brass grains & 5.142 & 14.500 & 8 \\
100 Al-5.2\% Sn alloy grains & 5.02 & 12.48 & 10 \\
Tetrakaidecahedron & 5.14 & 14.00 & \\
Pentagonal dodecahedron & 5.00 & 12.00 & \\
Cube & 4.00 & 6.00 & \\
\hline
\end{tabular}



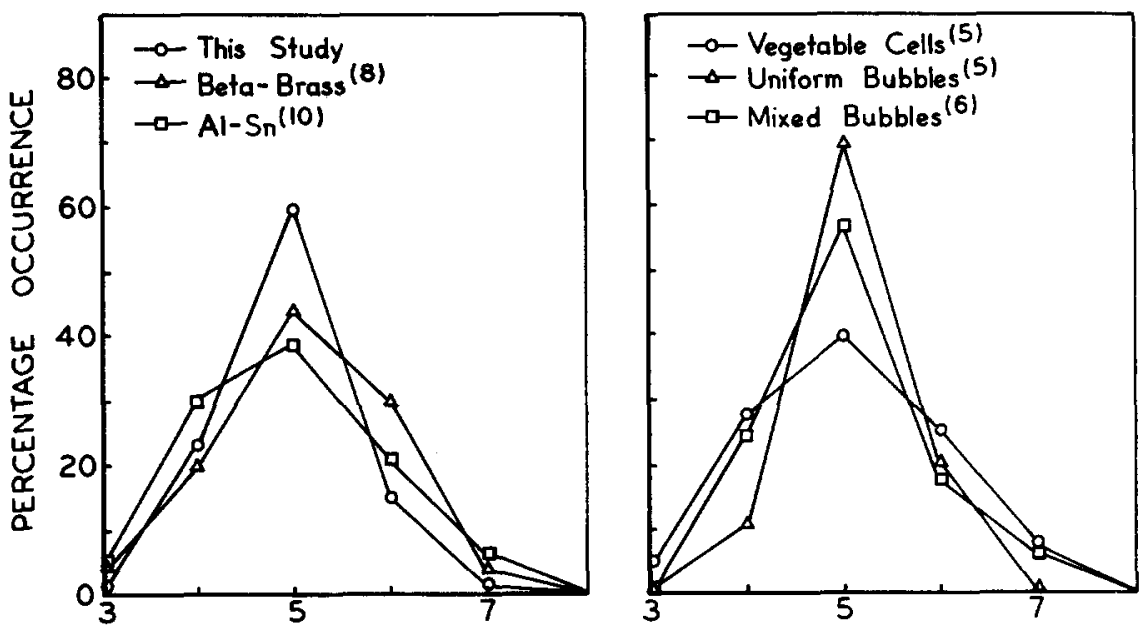

NUMBER OF EDGES PER FACE

FIG. 7. Distribution of edges per face for various materials.

as compared with the expected average values of 5.6915 and 6.000 , respectively, for those two shapes. The expected distributions from these two polyhedral shapes were compared statistically with experimental values. The fit was excellent for the pentagonal dodecahedron and poor for the tetrakaidecahedron.

\section{Selection of Structure Type}

Although the goal of these quantitative measurements is to obtain data which enable a "typing" of the structure toward one particular polyhedral shape, it is naive to assume that only one cell shape exists in such a foam, because many shapes go together to make up the volume. A typing of shape merely suggests that the distribution of forms can be averaged toward one given shape.

The preceding results of the cellular characteristics are summarized below and lead to the conclusion that the polyurethane foam structure is represented most closely by assuming a cellular shape of pentagonal dodecahedra:

\begin{tabular}{lcccc}
\hline & $\begin{array}{c}\text { Observed } \\
\text { average }\end{array}$ & $\begin{array}{c}\text { Expected } \\
\text { tetrakai- } \\
\text { decahedron }\end{array}$ & $\begin{array}{c}\text { Expected } \\
\text { pentagonal } \\
\text { dodecahedron }\end{array}$ & $\begin{array}{c}\text { Expected } \\
\text { cube }\end{array}$ \\
\hline Faces/polyhedron & 13.2 & 14.0 & 12.0 & 6.0 \\
Edges/faces of polyhedron & 4.88 & 5.14 & 5.00 & 4.00 \\
Edges/sectioned polygon & 5.68 & 6.00 & 5.69 & 4.00 \\
\hline
\end{tabular}




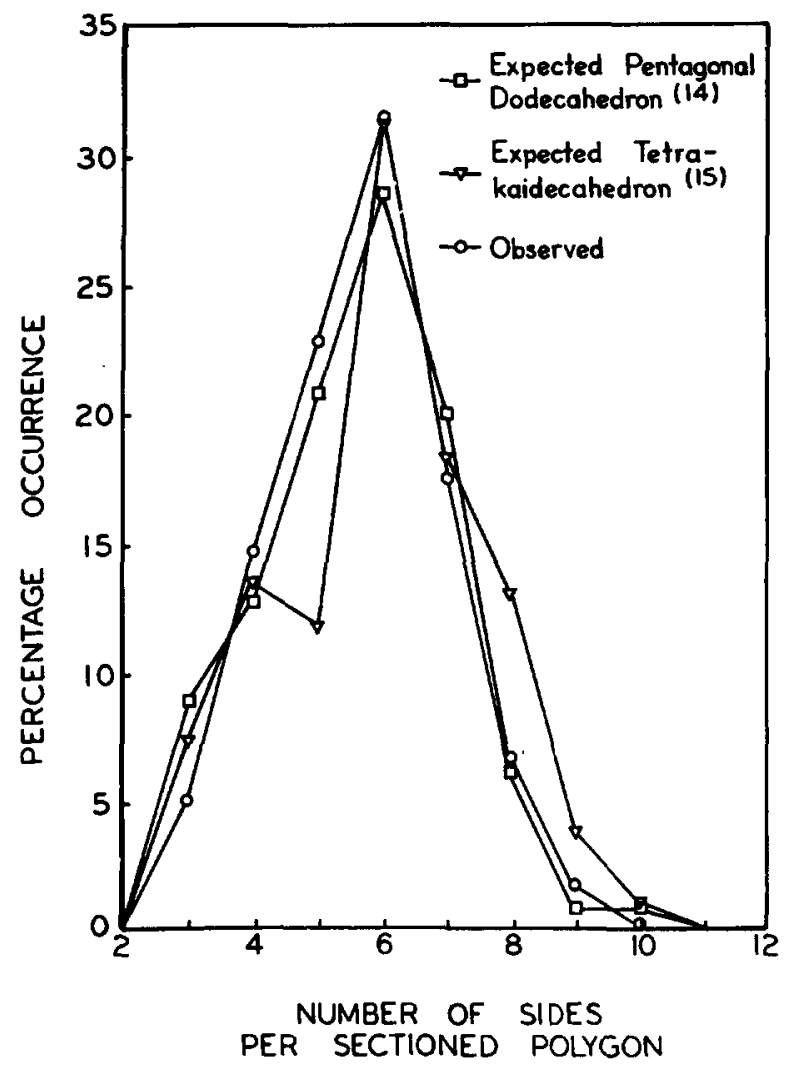

FIG. 8. Distribution of sides per sectioned polygon for various polyhedral shapes.

\section{Three-Dimensional Volume Distribution}

The measured volume distribution observed by filling 408 individual cells is shown in Fig. 9. Each data point is averaged with adjacent points for smoothness. These results yield an observed mean value of $0.0481 \mathrm{cc}$ and a standard deviation of $0.0179 \mathrm{cc}$. Also shown as a dark heavy line is a computed Gaussian distribution with a mean of $0.0477 \mathrm{cc}$ and standard deviation of $0.0188 \mathrm{cc}$. While such a fit cannot be exact because of discrepancies at the limits, it is concluded that the distribution of cell volumes within the foam is normal and can be described analytically by a Gaussian function.

\section{Review of Hull and Houk's Analysis}

Hull and Houk ${ }^{14}$ investigated in detail the distributions of the number of sides and the areas of "grains" in planar sections of various regular polyhedra by constructing wire models. Through proper orientation of the sectioning 


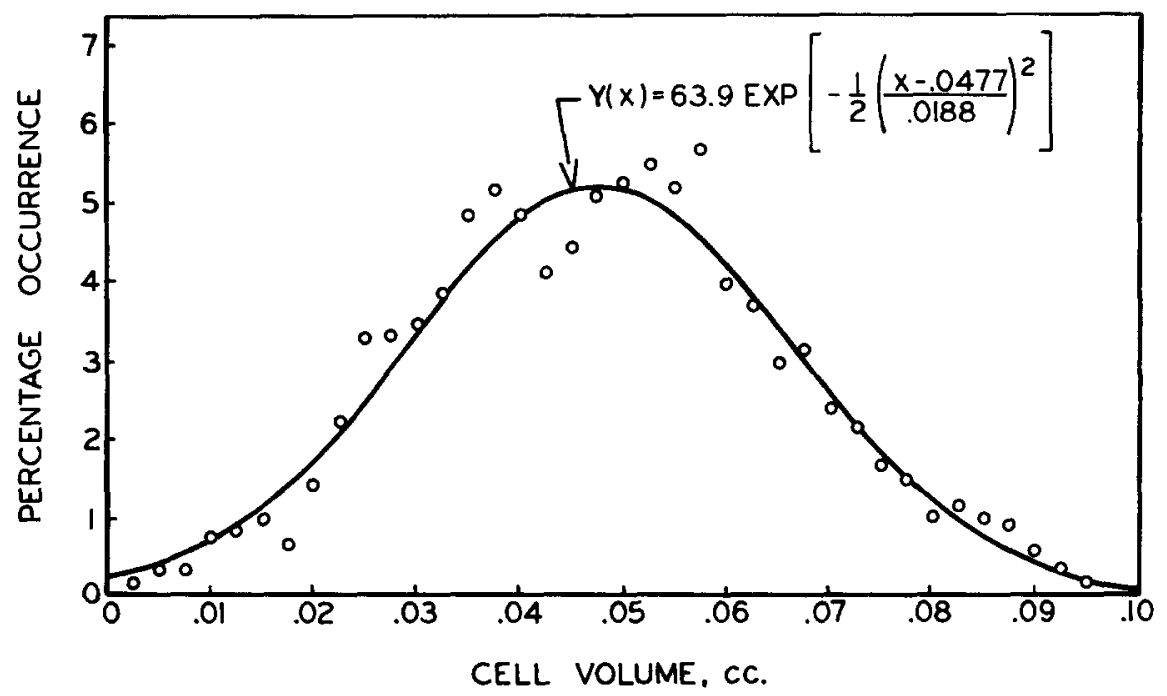

FIG. 9. Distribution of cell volumes.

plane, they selected a uniform distribution of orientations, roughly equivalent to a $5^{\circ}$ solid angle over the surface of a reference sphere. Characteristics of selected polyhedra of interest to this work are listed in Table II.

The results of Hull and Houk's measurements for the plane distribution curve

TABLE II

Characteristics of Selected Polyhedra*

\begin{tabular}{|c|c|c|c|c|}
\hline & Cube & Sphere & $\begin{array}{l}\text { Tetrakai- } \\
\text { decahedron }\end{array}$ & $\begin{array}{c}\text { Pentagonal } \\
\text { dodecahedron }\end{array}$ \\
\hline Volume & 1.0 & 1.0 & 1.0 & 1.0 \\
\hline Surface of figure & 6.00 & 4.83 & 5.31 & 5.30 \\
\hline$r$ of inscribed sphere & 0.50 & 0.62 & 0.54 & 0.56 \\
\hline $\begin{array}{l}\text { Average } r \\
\quad \text { (based on intersections) }\end{array}$ & 0.76 & 0.62 & 0.66 & 0.66 \\
\hline$r$ of circumscribed sphere & 0.87 & 0.62 & 0.70 & 0.71 \\
\hline $\begin{array}{l}\text { Average cross-section } \\
\text { area from distribution } \\
\text { curve, } A\end{array}$ & 0.66 & 0.81 & 0.77 & 0.78 \\
\hline $\begin{array}{l}\text { Maximum cross-section } \\
\quad \text { area, } A_{\max }\end{array}$ & 1.41 & 1.21 & 1.40 & 1.31 \\
\hline$A_{\max } / A$ & 2.14 & 1.50 & 1.83 & 1.69 \\
\hline
\end{tabular}

* From Hull and Houk. ${ }^{14}$ 

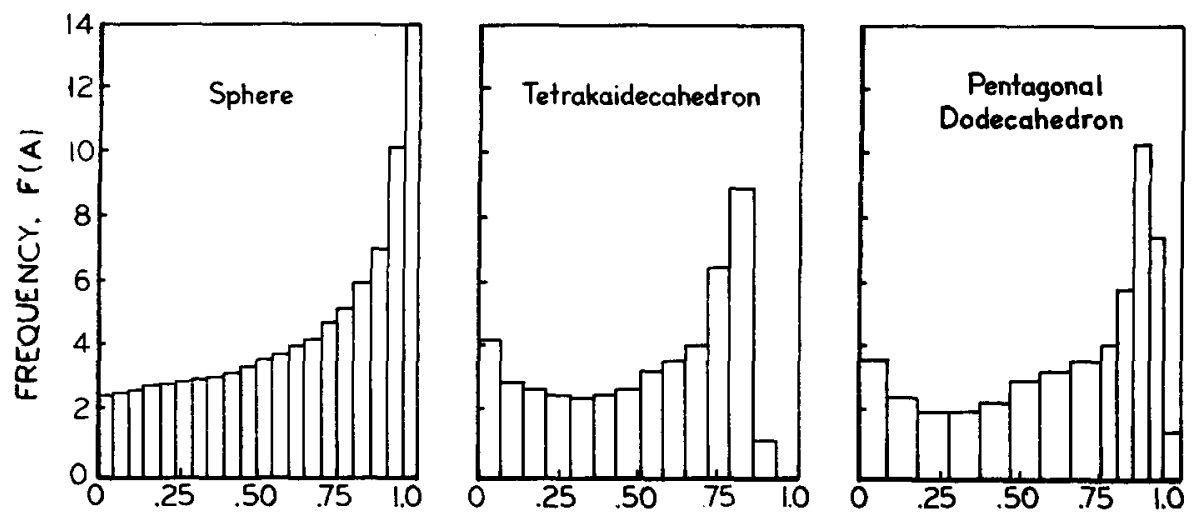

NORMALIZED AREA. A/A $\max$

FIG. 10. Distribution of normalized areas for various polyhedra. From Hull and Houk. ${ }^{14}$

of areas are shown for the sphere, tetrakaidecahedron, and pentagonal dodecahedron in Fig. 10. The latter two are of primary interest to this investigation. These plots are histograms of the frequency distribution of areas for each of the shapes. The area curves of polyhedra having more complex symmetry than the sphere are characterized by a rapid rise in frequency, $F(A)$, at small values of intersection. In addition, each curve has a large maximum between 0.7 and 0.9 for $A / A_{\max }$, while at the largest areas possible the curves reflect the differences in symmetry between the polyhedra.

The curves of the areal distributions represent the results of measurements from either of two viewpoints: (1) They may result from a statistically meaningful number of intersections of one given size of polyhedron, or (2) they may result from measurements of a volume containing identically sized polyhedra. Further, the shape of these curves is independent of the size of the polyhedra. That is, the same frequency distribution would be measured for a pentagonal dodecahedron, for example, whether very large or very small. These results pertain to only one particular size of polyhedra. The introduction of a volume containing a distribution of sizes is a complicating factor which is encountered in quantitative micrography.

These distribution curves take on special significance with relation to this investigation. With a knowledge of the areal distribution from a sectioning plane (two-dimensional data), and a knowledge of the volume distribution (threedimensional data), it is possible to calculate one of the distributions from the other with the aid of Hull and Houk's data. That is, using distribution of areas for one given polyhedral size and shape, and knowing the number and sizes within the volume under investigation, a calculated curve of areas for a section 
can be predicted and compared with actual measurements. This corresponds to a transformation of:

\section{3-dimensional (volume) $\rightarrow$ 2-dimensional (planar)}

\section{Calculation of Areal Distribution from Three-Dimensional Data}

The calculation of the two-dimensional area distribution curve was carried out in the following manner:(1) The measured volume distribution curve was analytically described by a Gaussian function having a mean of 0.0477 and a standard deviation of 0.0188 ; (2) this function was applied to the distribution curve of Hull and Houk, with the height of the Gaussian function becoming a multiplier, and the volume determining the value of $A_{\max }$ for each interval; (3) each calculated curve from (2) was normalized as to unit area under the curve; and finally (4) summation of the individual contributions of each curve was made across the axis for area. These summations as a function of area are a composite areal distribution curve which should be observed from a volume of foam having the cell volume distribution that was previously measured.

Calculations were made by computer for the sphere, pentagonal dodecahedron, and tetrakaidecahedron. Conversions from area to volume and vice versa for one size and shape were made using data from Table II and the assumption:

$$
V=K A_{\max }^{3 / 2}
$$

where $V$ is the volume, $K$ a constant, and $A_{\max }$ the maximum section area. Details of such calculations may be found elsewhere. ${ }^{21}$

The predicted areal distribution curve for the three shapes is shown in Fig. 11. The various polyhedra yield similar results as to position of the maxima and behavior toward large values of area. The major differences arise in the range of small areas, with the sphere distribution decreasing in the region of small areas, and the more complex shapes rising sharply near the origin. For each of the cases the peak height has been normalized to $100 \%$ at its maximum. The large values of small section areas predicted from the more complex polyhedra arise from sectioning planes intersecting corners of these bodies.

The results of the two-dimensional sectioning analysis are shown in Fig. 12 and compared with the predicted curves based on a shape assumption of the pentagonal dodecahedron and tetrakaidecahedron. Both the computer-generated curves based on the shape of the pentagonal dodecahedron and the tetrakaidehedron are in close agreement with the experimentally observed distribution. The experimental values at small section areas fit more closely the tetrakaidecahedron prototype, while for values larger than 0.2 in. $^{2}$ the pentagonal dodecahedron is preferred. A least-squares analysis indicates a better fit to the pentagonal dodecahedron. The direct comparison shows that an assumption of the sphere shape is not valid, particularly at small values of area. Thus, the 


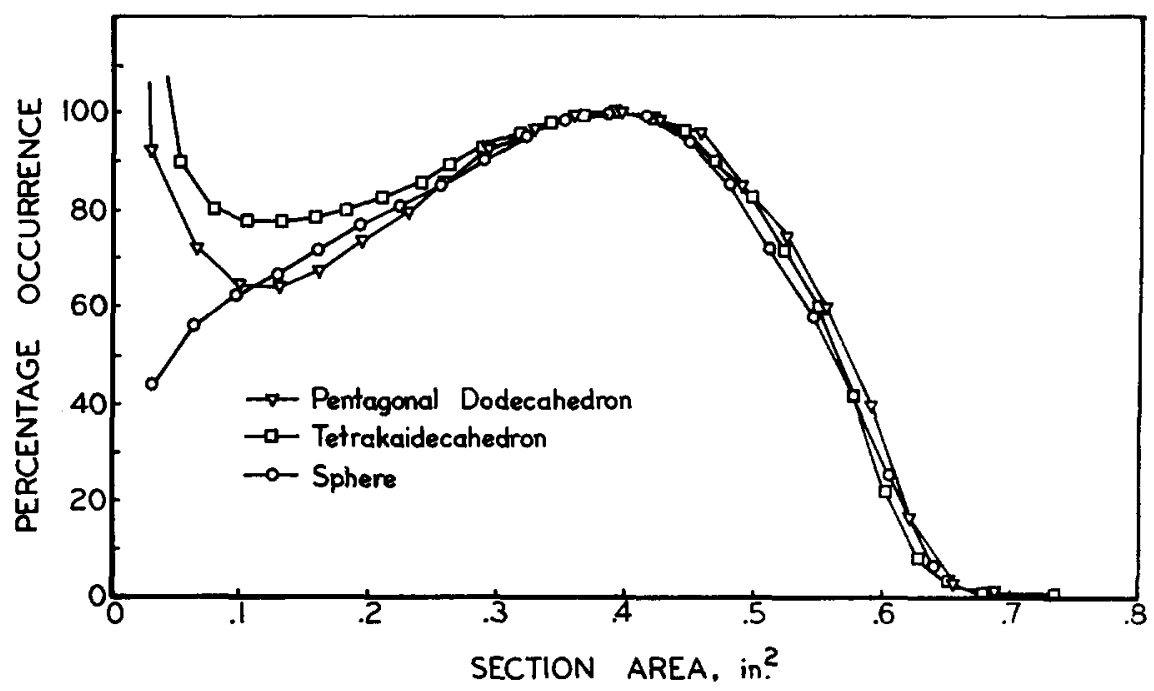

FIG. 11. Calculated distribution of section areas for various polyhedra.

generally accepted methods of volume distribution calculation utilized in quantitative microscopy would lead to erroneous results if applied to this system.

The results of the dimensional transformation coupled with the previous measurements of the cell characteristics demonstrate that the pentagonal dodecahedron is a reasonable prototype or model for foam cells. The selection

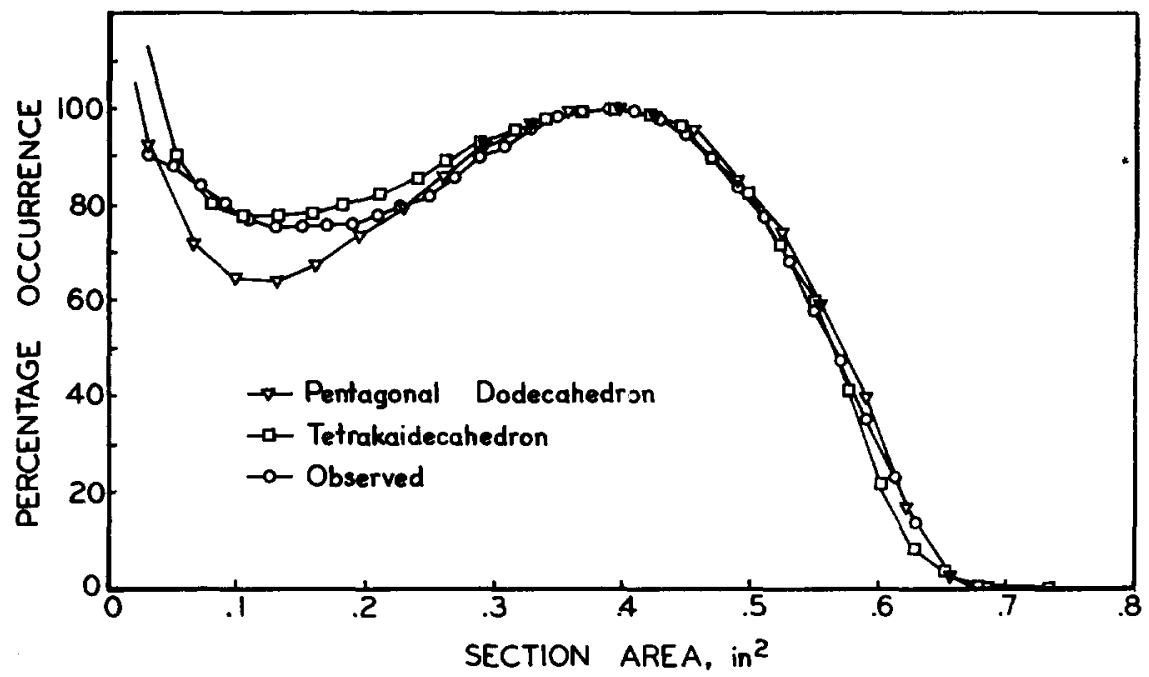

FIG. 12. Comparison of observed and calculated distributions of section areas. 
of this polyhedron as opposed to the space-filling tetrakaidecahedron arises most likely from the nonideality of the foam and the variation of cell shapes and volumes encountered in the material.

\section{Summary}

A commercial polyurethane foam having only a skeletonal structure was analyzed from both a two-dimensional and a three-dimensional standpoint. It was possible to determine characteristics of the form of these cells by considering cells within the foam as individual polyhedra. Comparison of these data to other cellular materials exhibits a strong similarity to data obtained from bubbles, vegetable cells, compressed balls, and alloys.

These distributions were compared to expected distributions from polyhedra having shapes that either completely or nearly fill all space while conforming to surface tension and topological requirements. On the basis of these data, the most probable shape of individual cells is that of the pentagonal dodecahedron, a 12-faced polyhedron with all faces being equal pentagons.

The distribution of areas from two-dimensional sectioning planes was observed on planar sections of polyester infiltrated foam and found to be close to a normal distribution. The distribution of volumes of cells was ascertained by filling individual cells with water and could be very closely described by a Gaussian function having a standard deviation of 0.40 of the mean volume $(0.0477 \mathrm{cc})$.

Using the Gaussian function describing the measured volume distribution and areal sectioning data for various polyhedral shapes existent in the literature, a predicted areal curve was numerically calculated based on selected particular shapes and the measured volume distribution. The calculated areal curves based on the shape for the pentagonal dodecahedron and tetrakaidecahedron fit the experimentally observed areal curve very closely. In contrast, the data show that an assumption of a spheroidal cell shape is invalid, particularly for small areas. Hence, calculations of volume distributions from observed area distributions established in the literature as accepted practice do not yield accurate results.

These results show that the most representative cellular shape for foam cells is the pentagonal dodecahedron, and present unusual data which permit for the first time a direct check of the validity of calculated areal distributions based on spatial volume measurements. Further, it is demonstrated that accurate predictions can be made of transformations between two-dimensional and three-dimensional data, providing characteristic cell shape and distribution can be adequately determined.

\section{References}

1. F. T. Lewis, Proc. Am. Acad. Arts Sci., 58 (1923) 537.

2. F. T. Lewis, Proc. Am. Acad. Arts Sci., 68 (1933) 251. 
3. J. W. Marvin, Am. J. Botany, 26 (1939) 487.

4. J. W. Marvin, Am. J. Botany, 26 (1939) 280.

5. E. B. Matzke, $A m$. J. Botany, 33 (1946) 58.

6. E. B. Matzke and J. Nestler, Am. J. Botany, 33 (1946) 130.

7. J. Plateau, Statique Experimental et Theorique des Liquides, Ghent, 1873.

8. C. H. Desch, J. Inst. Metals, 22 (1919) 241.

9. E. Scheil and H. Wurst, Z. Metallk., 28 (1936) 340.

10. W. M. Williams and C. S. Smith, Trans. AIME, 194 (1952) 755.

11. Lord Kelvin, Phil. Mag., 24 (1887) 503.

12. C. S. Smith, Sci. Am., 190 (1954) 58.

13. D. McLean, Grain Boundaries in Metals, Oxford Press, London, 1957.

14. F. C. Hull and W. J. Houk, Trans. AIME, 197 (1953) 565.

15. E. J. Myers, Ph.D. Thesis, University of Michigan, 1961.

16. C. S. Smith, Metal Interfaces, American Society for Metals, Cleveland, 1952, p. 65.

17. E. E. Underwood, in Quantitative Microscopy (DeHoff and Rhines, eds.), McGrawHill, New York, 1968, p. 149.

18. F. Schuckher, in Quantitative Microscopy (DeHoff and Rhines, eds.), McGraw-Hill, New York, 1968, p. 201.

19. H. B. Aaron, R. D. Smith, and E. E. Underwood, Paper No. 16, First International Conference for Sterology; Vienna, Austria, 1963.

20. C. S. Smith, Met. Rev., 9 (1964) 1.

21. P. L. White, Ph.D. Thesis, University of Michigan, 1969.

Accepted April 6, 1970 